\title{
Chemoselective Hydrogenation of $-\mathrm{C}=\mathrm{O}$ Bond in Multiple Unsaturated Compounds Over Metals Supported on Mesoporous Materials
}

\author{
Irina L. Simakova*a,b, Yuliya S. Demidova ${ }^{a, b}$, \\ Andrey V. Simakov and Dmitry Yu. Murzin ${ }^{\mathrm{d}}$ \\ ${ }^{a}$ Boreskov Institute of Catalysis SB RAS \\ 5 Akademika Lavrentieva, Novosibirsk, 630090, Russia \\ ${ }^{b}$ Novosibirsk State University \\ 2 Pirogova Str., Novosibirsk, 630090, Russia \\ 'Universidad Nacional Autónoma de México \\ Centro de Nanociencias y Nanotecnología, \\ km. 107 carretera Tijuana a Ensenada \\ Ensenada, Baja California, C.P. 22860, México \\ 'Process Chemistry Centre, Åbo Akademi University \\ Turku/Abo, FI-20500, Finland
}

Received 25.09.2019, received in revised form 10.10.2019, accepted 20.11.2019

\begin{abstract}
Liquid-phase hydrogenation of crotonaldehyde in non-polar and polar solvents was studied on monometallic $\mathrm{Pd}$, Pt, Ir, Re catalysts using mesoporous Sibunit and $\mathrm{Al}_{2} \mathrm{O}_{3}$ as supports. In the presence of $P d$ the $-C=C$ - bond of crotonaldehyde was preferably hydrogenated to form butanal, while butanal and crotyl alcohol are formed over Ir catalysts. Crotonaldehyde hydrogenation in 1,4-dioxane did not exhibit further butanal to butanol hydrogenation. Application of Re as a catalyst leads to formation of crotyl alcohol, with activity being, however, twofold lower than of Ir catalysts. Pt/C is almost inactive in the hydrogenation of crotonaldehyde. Formation of crotyl alcohol occurs most efficiently over Ir/Al $\mathrm{O}_{3}$ in aprotic nonpolar solvents (decane), with selectivity to crotyl alcohol increasing with temperature showing $30 \%$ at $25 \%$ conversion under $180{ }^{\circ} \mathrm{C}$ and hydrogen pressure $0.84 \mathrm{MPa}$. Crotonaldehyde hydrogenation in a polar protonic solvent (ethanol) results in butanal acetalization.
\end{abstract}

Keywords: multiple unsaturated compounds, crotonaldehyde, crotyl alcohol, competitive hydrogenation, $P d$, Pt, Ir, Re, alumina, carbon.

(c) Siberian Federal University. All rights reserved

* Corresponding author E-mail address: simakova@catalysis.ru 
Citation: Simakova I.L., Demidova Yu.S., Simakov A.V., Murzin D.Yu. Chemoselective hydrogenation of $-\mathrm{C}=\mathrm{O}$ bond in multiple unsaturated compounds over metals supported on mesoporous materials, J. Sib. Fed. Univ. Chem., 2019, 12(4), 536549. DOI: $10.17516 / 1998-2836-0149$.

\section{Хемоселективное гидрирование $-\mathrm{C}=\mathrm{O}$ группы \\ полиненасыщенных соединений \\ на металлических катализаторах, нанесенных \\ на мезопористые носители}

И.Л. Симакова ${ }^{a, \tilde{\sigma}}$, Ю.С. Демидова ${ }^{a, \tilde{\sigma}}$, А.В. Симаков ${ }^{\mathrm{B}}$, Д.Ю. Мурзин ${ }^{\mathrm{r}}$

аинститут катализа им. Г.К. Борескова СО РАН Россия, 630090, Новосибирск, пр. Академика Лаврентьева, 5

${ }^{6}$ Новосибирский государственный университет Россия, 630090, Новосибирск, ул. Пирогова, 2

${ }^{6}$ Центр нанонауки и нанотехнологии Национальный независимый университет Мексики

Мексика, 22860, Энсенада

`Кафедра промышленной химии и инженерии химических реакиий

Академия Або

Финляндия, 20500, Турку

Изучен прочесс жидкофазного гидрирования кротонового альдегида в неполярных и полярных средах на монометаллических Pd-, Pt-, Ir-, Re-катализаторах, нанесенных на мезопористые носители Сибунит и $\mathrm{Al}_{2} \mathrm{O}_{3}$. Показано, что в присутствии $\mathrm{Pd}$ предпочтительно гидрируется - $C=C$ - связь с образованием бутаналя, тогда как на Ir-катализаторах образуются бутаналь u кротиловый спирт. Применение Re приводит $\kappa$ образованию кротилового спирта, однако активность катализатора в 2 раза ниже, чем Ir. Pt/C малоактивен в гидрировании кротонового альдегида. Наиболее эффективно образование кротилового спирта происходит при гидрировании кротонового альдегида на $\mathrm{Ir} / \mathrm{Al}_{2} \mathrm{O}_{3}$ в апротонных неполярных растворителях (декан), при этом селективность по кротиловому спирту возрастает с температурой, достигая максимального значения 30 \% при конверсии $25 \%$, температуре 453 К и давлении водорода 0,84 МПа. При проведении реакиии в полярном протонном растворителе (этаноле) образуется больмое количество продуктов ачетализации.

Ключевые слова: полиненасыщенные соединения, кротоновый альдегид, кротиловый спирт,

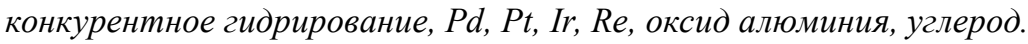




\section{Введение}

Реакции каталитического гидрирования промышленно важны и широко используются для создания продуктов нефтехимии, фармацевтики, пищевой промышленности, производства агрохимикатов и топлив [1]. Селективное гидрирование чрезвычайно важно для синтеза ряда полезных продуктов в различных областях, таких как полимеры, парфюмерия и фармацевтические препараты [2]. В пищевой промышленности полиненасыщенные жидкие растительные масла гидрируют в мононенасыщенные жиры, повышая тем самым химическую стабильность [3-5]. Хемоселективное гидрирование $\alpha, \beta$-ненасыщенных альдегидов и кетонов в ненасыщенные спирты представляет особый интерес, поскольку ненасыщенные спирты являются ценными соединениями для различных отраслей промышленности, таких как фармацевтика, парфюмерия и производство косметических средств. Например, коричный спирт применяется в синтезе антибиотика хлоромицетина, кротиловый спирт (КС) используется для синтеза витамина Е, пищевых консервантов (сорбиновая кислота), антибиотика левомицетина и других соединений [6]. КС получают селективным гидрированием кротонового альдегида (КА), превращение которого может протекать по двум маршрутам - через промежуточное гидрирование $-\mathrm{C}=\mathrm{O}$ или $-\mathrm{C}=\mathrm{C}-$ связи, при этом полное гидрирование КА приводит к одному и тому же продукту - бутанолу (рис. 1).

Многие исследования показали, что металлы VIII группы имеют специфическую селективность к ненасыщенным спиртам: иридий и осмий довольно селективны; палладий, родий и никель являются неселективными или малоселективными; платина, рутений и кобальт умеренно селективны. Эти тенденции были подтверждены для гидрирования КА (Os $>\mathrm{Ir}>\mathrm{Ru}>$ $\mathrm{Rh}=\mathrm{Pt}=\mathrm{Pd})$, а также для гидрирования коричного альдегида (Os $>\mathrm{Ir}>\mathrm{Pt}>\mathrm{Ru}>\mathrm{Rh}>\mathrm{Pd})$ [3]. Было также показано, что на Pt может быть достигнута высокая селективность по отношению к ненасыщенным спиртам [7]. Одним из самых распространенных носителей для катализаторов гидрирования является $\mathrm{Al}_{2} \mathrm{O}_{3}$ [8], однако на монометаллических катализаторах, нанесенных на $\gamma-\mathrm{Al}_{2} \mathrm{O}_{3}$ и $\mathrm{SiO}_{2}$, образуются, главным образом, насыщенные альдегиды [9]. Установлено, что металлические катализаторы на основе оксидов металлов, обеспечивающих сильное взаимодействие металл-носитель, такие как $\mathrm{TiO}_{2}, \mathrm{ZrO}_{2}$ и $\mathrm{CeO}_{2}, \mathrm{FeOx}, \mathrm{SnOx}, \mathrm{ZnO}$ [1], $\mathrm{Ga}_{2} \mathrm{O}_{3}$ [9], модифицирующее электронные свойства активного компонента [7], становятся более активны и селективны, чем катализаторы на основе инертных оксидов, таких как $\mathrm{SiO}_{2}$. Вместе с тем недавние

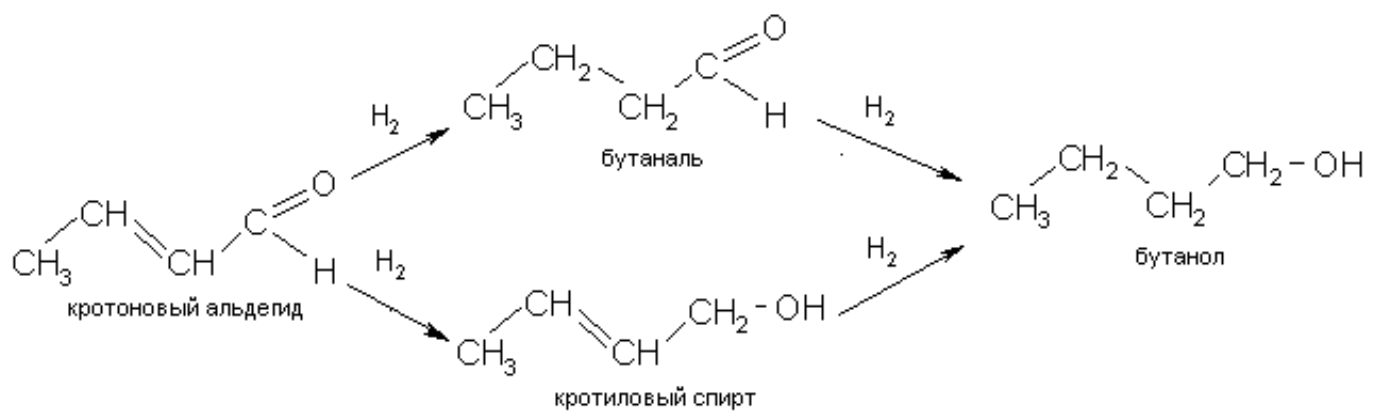

Рис. 1. Схема гидрирования КА

Fig. 1. Scheme of crotonaldehyde hydrogenation 
Таблица 1. Каталитическое гидрирование КА: катализаторы, условия реакции, данные по активности и селективности

Table 1. Catalytic hydrogenation of crotonaldehyde: catalysts, reaction conditions, conversion, selectivity

\begin{tabular}{|c|c|c|c|c|c|c|c|}
\hline \multirow[b]{2}{*}{ Катализатор } & \multicolumn{4}{|c|}{ Условия процесса } & \multirow{2}{*}{$\begin{array}{c}\text { Конверсия } \\
\text { КА, \% }\end{array}$} & \multirow{2}{*}{$\begin{array}{c}\text { Селект. по } \\
\text { КС, \% }\end{array}$} & \multirow[b]{2}{*}{ Литература } \\
\hline & Кат/КА, г/л & Растворитель & $\mathrm{T},{ }^{\circ} \mathrm{C}$ & $\begin{array}{l}\mathrm{P}_{\mathrm{H} 2}, \\
\mathrm{M \Pi а}\end{array}$ & & & \\
\hline $3,8 \% \mathrm{Ir} / \mathrm{TiO}_{2}$ & $3: 1$ & вода & 100 & 2 & 88 & 66 & {$[12]$} \\
\hline $3 \% \mathrm{Ir} / 0,05 \% \mathrm{Fe} / \mathrm{BN}$ & $100: 1$ & - & 80 & 0,1 & 55,6 & 84,4 & [13] \\
\hline $\mathrm{IrReOx} / \mathrm{SiO}_{2}$ & $50: 1$ & вода & 180 & 0,1 & 45,2 & 60,3 & {$[14]$} \\
\hline $\mathrm{Pt} / \mathrm{SiO}_{2}$ & $100: 1$ & $\begin{array}{c}\text { 2-метил-2- } \\
\text { бутанол }\end{array}$ & 100 & 0,1 & 93,5 & 1,3 & {$[15]$} \\
\hline $\mathrm{Pt} / \mathrm{ZnO}-175$ & $5: 2$ & $\begin{array}{c}\text { диоксан/ } \\
\text { вода/ } \mathrm{H}^{+} \\
\end{array}$ & 30 & 0,4 & 12 & 96 & {$[8]$} \\
\hline $5 \% \mathrm{Pd} / \mathrm{C}$ & $25: 1$ & метанол & 40 & 0,5 & 96,5 & 14,8 & {$[16]$} \\
\hline
\end{tabular}

исследования выявили, что и инертные носители с определенной мезопористой структурой могут обеспечивать высокую селективность по КС [10]. Так, селективность по КС на Ir/SiO $\mathrm{So}_{2}$ достигала 24 \% в зависимости от пористости носителя, тогда как селективность по КС на $\mathrm{Ir} / \mathrm{TiO}$ в этих условиях составляла $25 \%$ [10].

Исследование влияния условий реакции в жидкой фазе показало, что конверсия и селективность существенно зависят от давления водорода, температуры реакции [11] и типа растворителя [7]. Оценка эффектов растворителя особенно затруднена из-за отсутствия систематических экспериментальных данных, некоторые из них приведены в табл. 1.

Целью данной работы является изучение влияния природы металлов VIII группы $(\mathrm{Pd}, \mathrm{Pt}$, $\mathrm{Re}, \mathrm{Ir})$, нанесенных на мезопористые углеродный и алюмооксидный носители, а также типа растворителя (этанол, диоксан и декан) на селективность гидрирования $-\mathrm{C}=\mathrm{O}$ группы КА в условиях конкурентного гидрирования.

\section{Экспериментальная часть}

Реагенты. В работе использовали кротоновый альдегид (содержание транс-изомера 95 \%, ТУ 113-03-389-80), этанол (ГОСТ 17299-78), 1,4-диоксан (ГОСТ 10455-80), н-декан (ТУ 6-093614-74), азот (ГОСТ 9293-74), водород (ГОСТ 3022-80). В качестве прекурсоров активного компонента выступали следующие реагенты: палладий хлорид $\mathrm{PdCl}_{2}$ (ТУ 6-09-2025-84 Peaxим, г. Москва), рениевая кислота $\mathrm{HReO}_{4}$ (ТУ 38-301-41-137-90 Реахим, г. Москва), платинохлористоводородная 6-водная кислота $\mathrm{H}_{2} \mathrm{PtCl}_{6}$ (ТУ 2612-034-00205067-2003 ОАО АУРАТ, г. Москва), иридий (III) хлорид гидрат $\mathrm{IrCl}_{3}$ (ТУ 2625-067-00196533-2002 ОАО “Красноярский завод цветных металлов имени В.Н. Гулидова”, г. Красноярск), хлорид олова (ТУ 6-09-5393-88). Для приготовления катализаторов использовали носители: мезопористый углеродный материал «Сибунит» $\left(\mathrm{S}_{\text {уд }}=352 \mathrm{м}^{2} / \Gamma, \mathrm{V}_{\text {пор }}=0,4 \mathrm{~cm}^{3} / \Gamma\right.$, фракция 40-70 $\left.\mu \mathrm{M}\right)$ и порошкообразный $\gamma-\mathrm{Al}_{2} \mathrm{O}_{3}\left(\mathrm{~S}_{\text {уд }}=129 \mathrm{M}^{2} / \Gamma\right.$, $\left.\mathrm{V}_{\text {пор }}=0,2 \mathrm{~cm}^{3} / \Gamma, 40-63 \mu \mathrm{M}\right)$.

$$
-539-
$$


Очистку КА (температура кипения $104^{\circ} \mathrm{C}$ ) осуществляли вакуумной перегонкой под водоструйным насосом. Фракцию, отобранную при температуре $25-33{ }^{\circ} \mathrm{C}$, применяли для проведения экспериментов по гидрированию.

Методика приготовления катализаторов. Катализаторы для реакции гидрирования КА с массовым содержанием $4 \%$ - $\mathrm{Pd} / \mathrm{C}, \mathrm{Ir} / \mathrm{Al}_{2} \mathrm{O}_{3}, \mathrm{Re} / \mathrm{Al}_{2} \mathrm{O}_{3}$ - и 3 \% - Ir/C, Pt/C - были приготовлены методом пропитки носителя водным раствором соответствующего прекурсора: $\mathrm{H}_{2} \mathrm{PtCl}_{6}(0,1$ $\mathrm{M}), \mathrm{H}_{2} \mathrm{IrCl}_{6}(0,5 \mathrm{M}), \mathrm{H}_{2} \mathrm{PdCl}_{4}(0,1 \mathrm{M})$ или $\mathrm{HReO}_{4}(1 \mathrm{M})$. Перед нанесением оксид алюминия прокаливали на воздухе при температуре $520{ }^{\circ} \mathrm{C}$ в течение 3 ч. После пропитки образцы сушили 17 ч при температуре $109^{\circ} \mathrm{C}$, восстанавливали в $\mathrm{H}_{2}$ при температуре $400{ }^{\circ} \mathrm{C}$ в течение 3 ч (2 K/ мин).

Каталитические эксперименты. Эксперименты по жидкофазному гидрированию КА проводили в автоклаве (150 мл) из нержавеющей стали с перемешиванием 1000 об/мин при давлении водорода $0,55 \div 1,15$ МПа и температуре в этаноле и диоксане $333 \mathrm{~K}$, в декане 413-453 К. Начальная концентрация КА $\left(C_{0}\right)=0,6 \div 0,74 \mathrm{M}$, объем растворителя 15 мл.

Методика анализа компонентов реакции. Состав продуктов определяли методом газожидкостной хроматографии на хроматографе «Хромос» ГХ-1000 с пламенно-ионизационным детектором и кварцевой капиллярной колонкой (60 м / 0,25 мм / 0,25 мкм) ВР20 при температуpe $50-240{ }^{\circ} \mathrm{C}$ со скоростью нагрева $10 \mathrm{~K} /$ мин. Температура детектора и испарителя составляла $250{ }^{\circ} \mathrm{C}$. В качестве газа-носителя использовали водород со скоростью потока $20 \mathrm{~cm}^{3} /$ мин. Идентификацию конденсированных продуктов проводили методом хроматомасс-спектроскопии на приборах VG-7070 GC/MS (колонка 30 м х 0.2 мм Silicone SE-30) и Agilent 5973N EI/PCI (колонка 30 мм х 0,25 мм HР-5ms).

Селективность образования продукта определяли как отношение концентрации продукта к количеству превращенного КА. Конверсию КА рассчитывали как отношение концентрации прореагировавшего КА к его начальной концентрации.

\section{Физико-химические методы анализа катализаторов}

Определение процентного содержания металла в образцах катализаторов проводили рентгеноспектральным методом на флуоресцентном анализаторе VRA-30 c Cr-анодом рентгеновской трубки.

Исследования образцов методом просвечивающей электронной микроскопии (ПЭМ) осуществляли на просвечивающем электронном микроскопе высокого разрешения «JЕM-2010» (Япония) с разрешением по решетке 0,14 нм и ускоряющим напряжением 200 кВ.

Исследования методом ТГ-ДТА были выполнены на приборе Netzsch STA 409 РС. Измерения проводили с использованием корундовых тиглей. После загрузки образца около 30 мг и установки тигля на держатель температуру поднимали до $50^{\circ} \mathrm{C}$ и выдерживали 1 ч. Термические кривые восстановления образцов были записаны в диапазоне от 50 до $700{ }^{\circ} \mathrm{C}$ со скоростью нагрева 5 К/мин в смеси водорода и аргона. Скорости потоков водорода и аргона были одинаковыми - 20 мл/мин. Полученные данные были обработаны с помощью программы Netzsch Proteus Analysis и затем совмещены с данными масс-спектрометра в программе OriginPro 7.5G. 


\section{Результаты и обсуждение}

Исследование катализаторов физико-химическими методами

Гидрирование КА проводили в присутствии катализаторов после их предварительного восстановления согласно ранее разработанным методикам гидрирования различных органических субстратов [5, 17-20]. В соответствии с полученными данными ТПВ были определены оптимальные условия высокотемпературной восстановительной обработки для катализаторов $\mathrm{Pd} / \mathrm{C}, \mathrm{Pt} / \mathrm{C}, \mathrm{Ir} / \mathrm{C}, \mathrm{Ir} / \mathrm{Al}_{2} \mathrm{O}_{3}$ и $\mathrm{ReOx} / \mathrm{Al}_{2} \mathrm{O}_{3}$ (рис. 2).

Согласно данным ТГ-ДТА, термообработка образцов, синтезированных нанесением прекурсоров соответствующих металлов на углеродный носитель и оксид алюминия, сопровождается эндотермическими и экзотермическим эффектами, протекающими с изменением массы образцов (рис. 2). При нагревании в потоке водорода до $750{ }^{\circ} \mathrm{C}$ при взаимодействии водорода с прекурсорами металлов наблюдается максимум потери массы для $\mathrm{IrCl}_{3} / \mathrm{Al}_{2} \mathrm{O}_{3}$ при $171,2{ }^{\circ} \mathrm{C}$, для $\mathrm{HReO}_{4} / \mathrm{C}$ при $222,2{ }^{\circ} \mathrm{C}$ и для $\mathrm{H}_{2} \mathrm{PtCl}_{6} / \mathrm{C}$ при $186,4{ }^{\circ} \mathrm{C}[21]$ и с общим уменьшением массы образцов на 9,00, 2,57 и 2,85 вес. \% соответственно. Наибольшая потеря массы для $\mathrm{IrCl}_{3} / \mathrm{Al}_{2} \mathrm{O}_{3}$ связана, по-видимому, с удалением кристаллической воды из структуры соли. На кривой термического анализа для $\mathrm{HReO}_{4} / \mathrm{C}$ присутствие небольшого экзотермического эффекта обусловлено, видимо, взаимодействием оксида рения с водородом. Исследование методом ПЭМ показало, что образцы характеризуются равномерным распределением частиц по носителю, причем средний размер частиц $\mathrm{d} \sim 0,7 \div 2$ нм характерен для всех образцов (рис. 3 , показано для $\mathrm{Pt} / \mathrm{C})$.

Методом РФЭС (не показано) было установлено, что активные компоненты катализаторов $\mathrm{Pd} / \mathrm{C}, \mathrm{Pt} / \mathrm{C}, \mathrm{Ir} / \mathrm{C}, \mathrm{Ir} / \mathrm{Al}_{2} \mathrm{O}_{3}$ и $\mathrm{Re} / \mathrm{Al}_{2} \mathrm{O}_{3}$ представляют металлические частицы с незначитель-

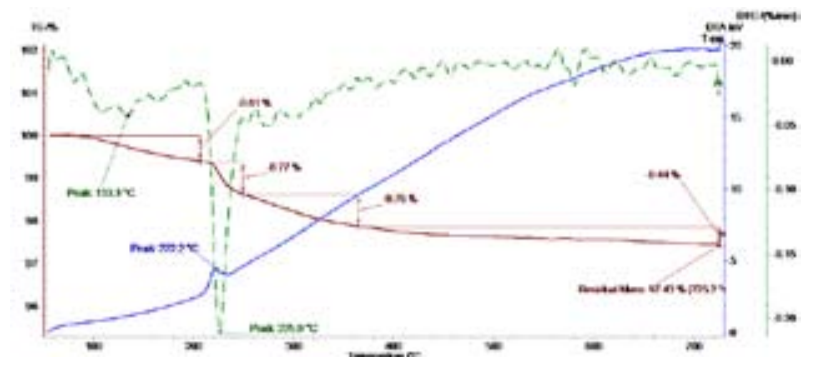

a

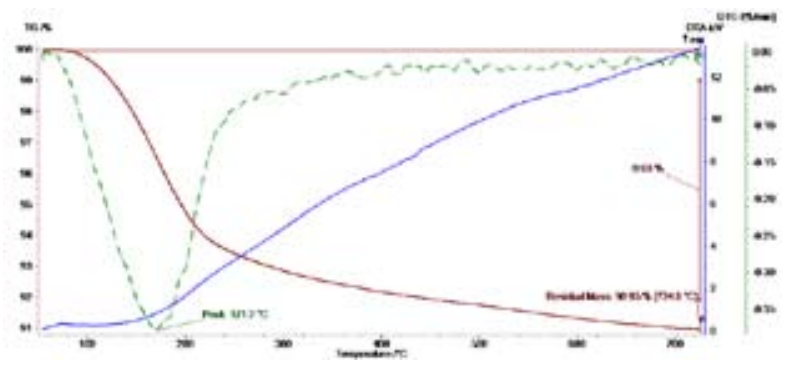

6

Рис. 2. Термические кривые ТГ-ДТА: a - $\mathrm{IrCl}_{3} / \mathrm{Al}_{2} \mathrm{O}_{3} ; \sigma-\mathrm{HReO}_{4} / \mathrm{Al}_{2} \mathrm{O}_{3}$

Fig. 2. DTA curves of $\mathrm{a}-\mathrm{IrCl}_{3} / \mathrm{Al}_{2} \mathrm{O}_{3}, \mathrm{~b}-\mathrm{HReO}_{4} / \mathrm{Al}_{2} \mathrm{O}_{3}$

$$
-541-
$$



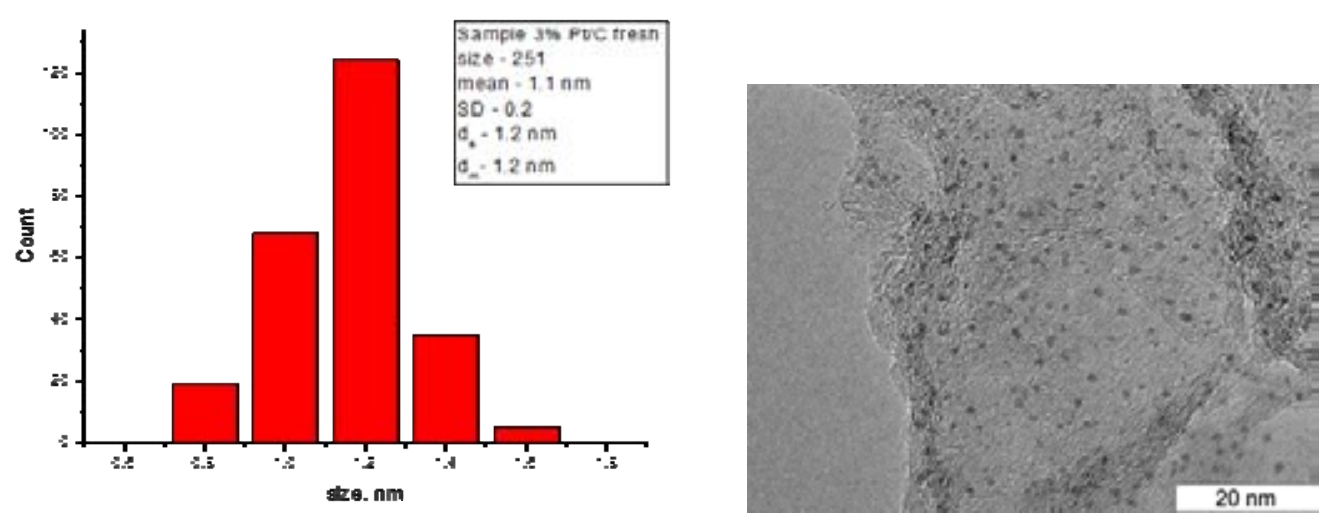

Рис. 3. Данные ПЭМ для образца $\mathrm{Pt} / \mathrm{C}$, полученного восстановлением $\mathrm{H}_{2} \mathrm{PtCl}_{6} / \mathrm{C}$ в $\mathrm{H}_{2}$

Fig. 3. TEM data for $\mathrm{Pt} / \mathrm{C}$ catalyst prepared by reduction of $\mathrm{H}_{2} \mathrm{PtCl}_{6} / \mathrm{C}$ in $\mathrm{H}_{2}$

ной долей окисленного металла за счет реокисления на воздухе, за исключением $\operatorname{Ir} / \mathrm{Al}_{2} \mathrm{O}_{3}$ и $\mathrm{Re} /$ $\mathrm{Al}_{2} \mathrm{O}_{3}$, в которых окисленное состояние металла немного выше, что обусловлено, по-видимому, влиянием носителя, содержащего подвижный кислород. Можно предположить, что в восстановительных условиях реакции частично окисленная фаза активного компонента претерпевает полное восстановление.

\section{Влияние природы металла и условий реакциии}

Первая серия экспериментов по гидрированию КА была проведена на катализаторе $\mathrm{Pd} / \mathrm{C}$ (рис. 4) с целью определения диапазона условий реакции для осуществления гидрирования КА в КС с заметной скоростью. Согласно данным ГЖХ-анализа, основными продуктами реакции гидрирования КА в этаноле являются 1,1-диэтоксибутан, бутаналь и бутанол. В продуктах реакции не был обнаружен КС, что соответствует термодинамическим расчетам, показывающим, что гидрирование связи - $\mathrm{C}=\mathrm{C}$ - представляет собой более благоприятный процесс по сравнению с гидрированием связи $-\mathrm{C}=\mathrm{O}$ с точки зрения термодинамики, так как разница в стандартной энергии Гиббса составляет 35 кДж/моль [7]. На рис. 4 изображено распределение концентраций продуктов от времени реакции при варьировании температуры от 333 до 373 К (рис. 4a) и давления водорода от 0,55 до 1,15 МПа (рис. 4б). Анализ кинетических кривых гидрирования КА указывает на слабую зависимость скорости реакции гидрирования от температуры и давления водорода (рис. 4).

Дальнейшее превращение бутаналя на $\mathrm{Pd} / \mathrm{C}$ в полярном протонном растворителе протекает по двум маршрутам: (i) гидрирование бутаналя в бутанол на Pd, (ii) нуклеофильное присоединение молекул этанола с образованием диэтилбутираля на кислых центрах на поверхности Сибунита [22] (рис. 5).

Важно отметить, что варьирование температуры и давления водорода в присутствии Рdкатализатора не позволяет направить гидрирование КА по маршруту образования КС. Гидрирование КА на катализаторе $\mathrm{Pt} / \mathrm{C}$ (рис. 6б) протекает со значительно более низкой скоростью, чем на $\mathrm{Pd} / \mathrm{C}$, наблюдается образование бутаналя и бутанола, но не КС. Отсутствие ацеталя при этом, по-видимому, связано с низкой степенью превращения КА. 


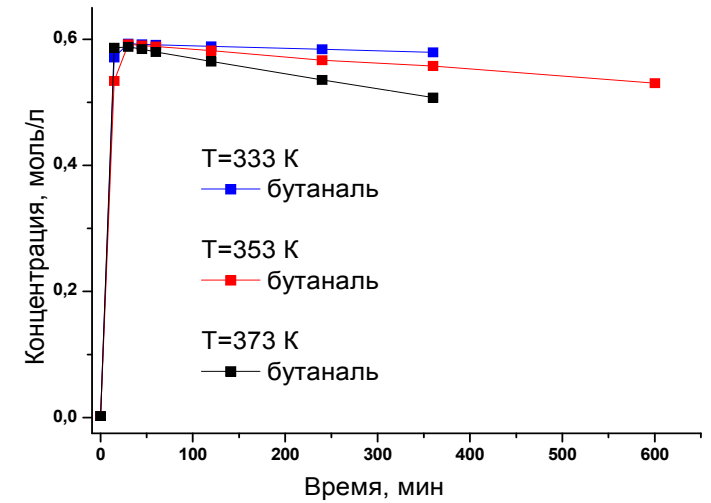

a)

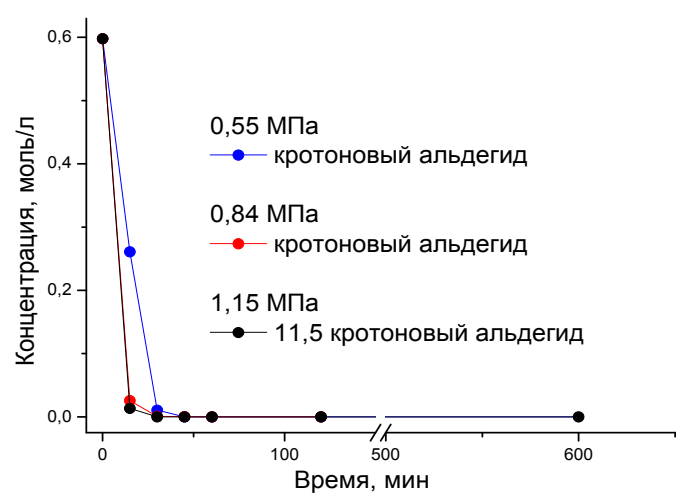

б)

Рис. 4. Кинетические кривые гидрирования КА в присутствии $\mathrm{Pd} / \mathrm{C}$ : a - влияние температуры реакции; б - влияние давления водорода. Условия реакции: $m_{\text {кат }}=10 \mathrm{Mг,} C_{0}=0,6 \mathrm{M}$, растворитель: этанол

Fig. 4. Kinetics of crotonaldehyde hydrogenation over $\mathrm{Pd} / \mathrm{C}$ a - effect of the reaction temperature on butanal formation, $\sigma$ - effect of hydrogen pressure on crotonaldehyde consumption. Reaction conditions: $m_{\text {cat }}=10 \mathrm{mg}$, $C_{0}=0.6 \mathrm{M}$, solvent: ethanol

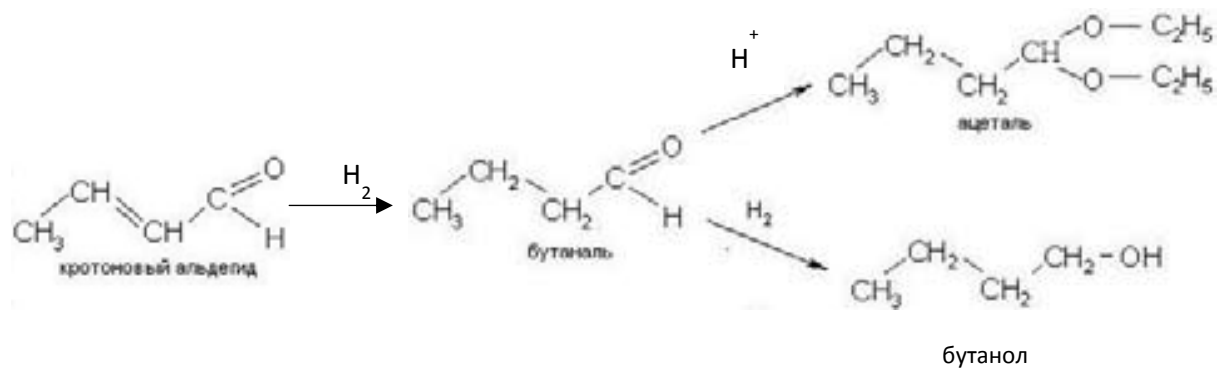

Рис. 5. Схема реакции гидрирования КА на $\mathrm{Pd} / \mathrm{C}$ в этаноле

Fig. 5. Scheme of crotonaldehyde hydrogenation over $\mathrm{Pd} / \mathrm{C}$ in ethanol

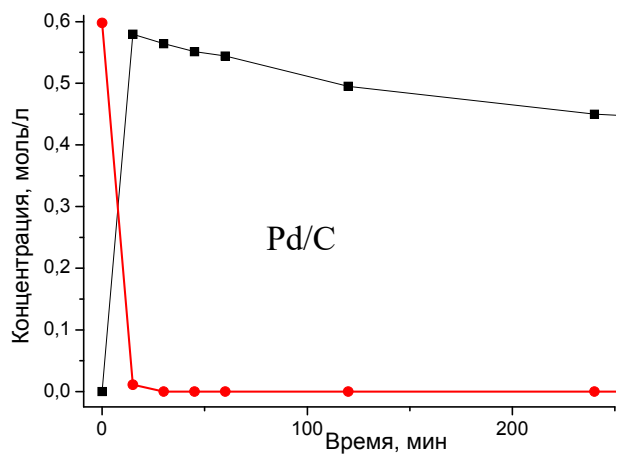

a)

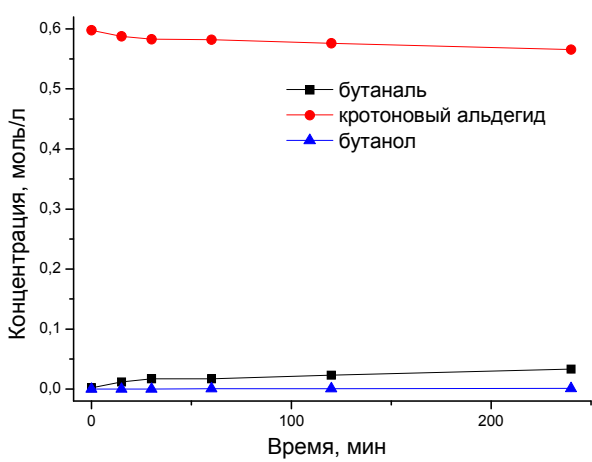

б)

Рис. 6. Кинетические зависимости для реакции гидрирования КА в присутствии: $\mathrm{a}-4$ \% $\mathrm{Pd} / \mathrm{C} ; 6-3 \% \mathrm{Pt} / \mathrm{C}$. Условия реакции: $m_{\text {кат }}=10 \mathrm{Mг}, T=333 \mathrm{~K}, P\left(\mathrm{H}_{2}\right)=0,55 \mathrm{MПа}, C_{0}=0,6 \mathrm{M}$, растворитель: этанол

Fig. 6. Kinetics of crotonaldehyde hydrogenation over; a $-4 \% \mathrm{Pd} / \mathrm{C} ; \sigma-3 \% \mathrm{Pt} / \mathrm{C}$. Reaction conditions: $m_{\text {cat }}=10 \mathrm{mg}$, $T=333 \mathrm{~K}, P\left(\mathrm{H}_{2}\right)=0.55 \mathrm{MPa}, C_{0}=0.6 \mathrm{M}$, solvent: ethanol 
Для систематизации данных и установления эффекта растворителя на ход реакции гидрирования были проведены эксперименты по гидрированию КА на 4 \% $\mathrm{Pd} / \mathrm{C}$ в малополярном апротонном растворителе - 1,4-диоксане. Показано, что проведение реакции в 1,4-диоксане не ведет к образованию КС и бутанола, но позволяет избежать ацетализации бутаналя (рис. 7). Проведение реакции гидрирования КА на катализаторе $\mathrm{Ir} / \mathrm{C}$ в $н$-декане (рис. $8 a$ ) позволило синтезировать целевой продукт - КС - и избежать образования побочных продуктов. По-видимому, апротонные неполярные растворители являются более эффективными в реакции гидрирования ненасыщенных карбонильных соединений, обеспечивая преимущественное получение продуктов полного и неполного гидрирования без последующей ацетализации. Кроме того, использование высококипящего н-декана $\left(\right.$ Ткип $\left.=174{ }^{\circ} \mathrm{C}\right)$ позволяет проводить исследование гидрирования КА в широком диапазоне температур и давлений водорода.

Таким образом, было установлено, что в присутствии $\mathrm{Pd} / \mathrm{C}$ предпочтительно гидрируется $-\mathrm{C}=\mathrm{C}$ - связь с образованием бутаналя, не затрагивая $-\mathrm{C}=\mathrm{O}$ связь, катализатор $\mathrm{Ir} / \mathrm{C}$ менее активен, однако более селективен в реакции гидрирования $-\mathrm{C}=\mathrm{O}$ группы, обеспечивая образование КС в заметных количествах, a Pt/C мало активен в данных условиях гидрирования.

Для изучения влияния природы мезопористого носителя на скорость реакции гидрирования КА и селективность образования КС была исследована активность Ir катализаторов на основе Сибунита и $\gamma-\mathrm{Al}_{2} \mathrm{O}_{3}$. Основным продуктом гидрирования КА в присутствии катализатоpa Ir/C является бутаналь, при этом наблюдается образование КС и бутанола в сопоставимых количествах (рис. $8 a$ ), в то время как на $\mathrm{Ir} / \mathrm{Al}_{2} \mathrm{O}_{3}$ основным продуктом является КС, образование бутаналя происходит с меньшей скоростью (рис. 8б). Селективность образования КС на $\mathrm{Ir} / \mathrm{Al}_{2} \mathrm{O}_{3}$ гораздо выше, чем на $\mathrm{Ir} / \mathrm{C}$, при этом максимальная селективность по КС наблюдается при одинаковой конверсии 20-25\%, достигая около 50 \% и $35 \%$ на $\mathrm{Ir} / \mathrm{Al}_{2} \mathrm{O}_{3}$ и $\mathrm{Ir} / \mathrm{C}$ соответственно (рис. 8в). На основании полученных данных можно сделать вывод, что мезопористый

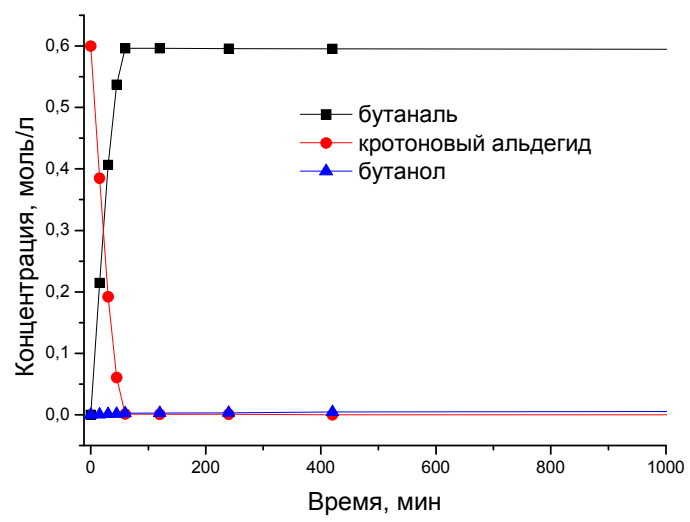

Рис. 7. Кинетические зависимости для реакции гидрирования КА на $4 \% \mathrm{Pd} / \mathrm{C}$ в 1,4- диоксане. Условия реакции: $m_{\text {кам }}(4 \% \mathrm{Pd} / \mathrm{C})=40 \mathrm{мг}, P\left(\mathrm{H}_{2}\right)=0,84 \mathrm{MПа}, T=323 \mathrm{~K}$

Fig. 7. Kinetics of crotonaldehyde hydrogenation over $4 \% \mathrm{Pd} / \mathrm{C}$ in 1,4-dioxane. Reaction conditions: $m_{\text {cat }}=40 \mathrm{mg}$, $T=323 \mathrm{~K}, P\left(\mathrm{H}_{2}\right)=0.84 \mathrm{MPa}, C_{0}=0.6 \mathrm{M}$ 


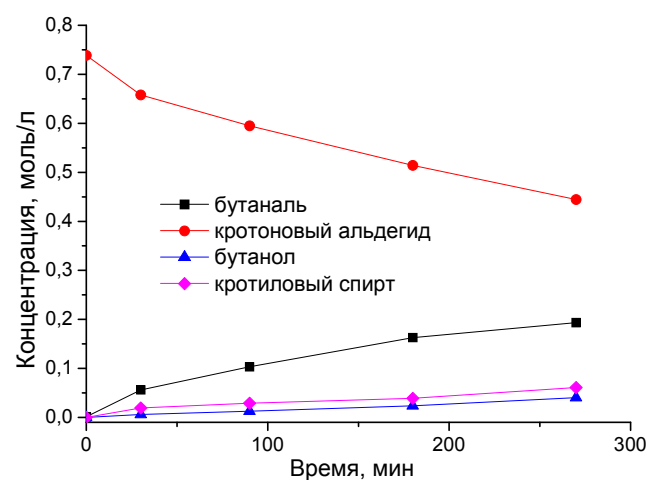

a)

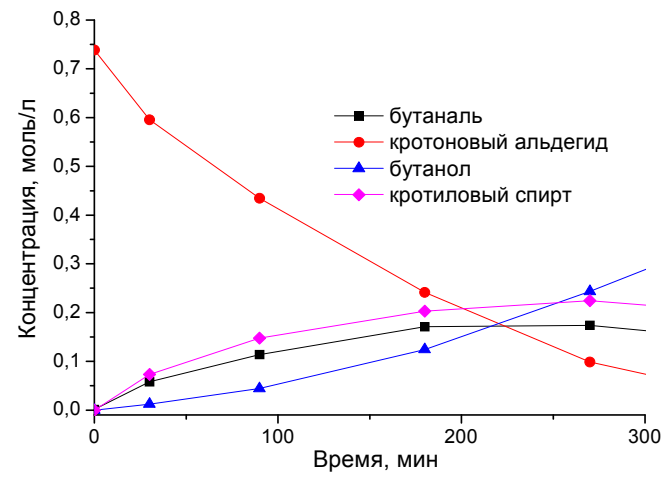

б)

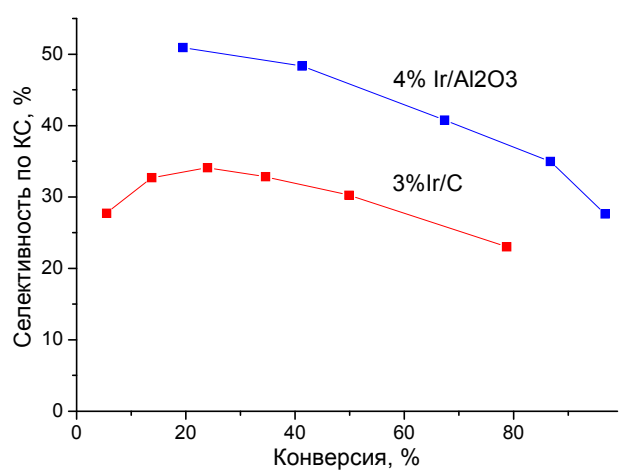

в)

Рис. 8. Кинетические зависимости для реакции гидрирования КА в присутствии $\mathrm{Ir}$, нанесенного на: $\mathrm{a}$ - углеродный носитель Сибунит; б - $\gamma-\mathrm{Al}_{2} \mathrm{O}_{3}$; в - зависимость селективности по КС от конверсии КА. Условия реакций: $m_{\text {кат }}=150$ мг, $P\left(\mathrm{H}_{2}\right)=1,15 \mathrm{MПа}(\mathrm{Ir} / \mathrm{C}), P\left(\mathrm{H}_{2}\right)=0,84 \mathrm{MПa}\left(\operatorname{Ir} / \mathrm{Al}_{2} \mathrm{O}_{3}\right), T=453 \mathrm{~K}, C_{0}=0,74$ М, растворитель: декан

Fig. 8. Kinetics of crotonaldehyde hydrogenation over Ir on a - carbon support Sibunit, $\sigma-\gamma-\mathrm{Al}_{2} \mathrm{O}_{3}, \mathrm{~B}-$ dependence of selectivity to crotyl alcohol vs. crotonaldehyde conversion for $\mathrm{Ir} / \mathrm{C}$ and $\mathrm{Ir} / \mathrm{Al}_{2} \mathrm{O}_{3}$. Reaction conditions: $m_{\text {cat }}=150$ $\mathrm{mg}, P\left(\mathrm{H}_{2}\right)=1.15 \mathrm{MPa}(\mathrm{Ir} / \mathrm{C})$ and $P\left(\mathrm{H}_{2}\right)=0.84 \mathrm{MPa}\left(\mathrm{Ir} / \mathrm{Al}_{2} \mathrm{O}_{3}\right), T=453 \mathrm{~K}, C_{0}=0,74 \mathrm{M}$, solvent: decane

$\gamma-\mathrm{Al}_{2} \mathrm{O}_{3}$ может быть использован в качестве носителя активного компонента в конкурентном гидрировании $-\mathrm{C}=\mathrm{O}$ группы. По-видимому, адсорбция $\mathrm{KA}$ на $\mathrm{Ir} / \mathrm{Al}_{2} \mathrm{O}_{3}$ происходит преимущественно по - $\mathrm{C}=\mathrm{O}$ группе, в то время как на $\mathrm{Ir} / \mathrm{C}$ и по $-\mathrm{C}=\mathrm{C}$ - связи.

Ранее авторами была показана высокая активность и селективность рениевых катализаторов в реакции гидрирования пентановой кислоты в пентанол [23], обусловленные высокой оксифильностью рения. Гидрирование КА в присутствии $\mathrm{Re} / \mathrm{Al}_{2} \mathrm{O}_{3}$ и $\mathrm{Ir} / \mathrm{Al}_{2} \mathrm{O}_{3}$ выявило, что оба металла обеспечивают образование КС, однако селективность по КС и скорость гидрирования КА в присутствии $\mathrm{Re} / \mathrm{Al}_{2} \mathrm{O}_{3}$ приблизительно в 2 раза ниже, чем на $\mathrm{Ir} / \mathrm{Al}_{2} \mathrm{O}_{3}$ (рис. $9 a, \sigma)$.

Установлено, что температура оказывает существенное влияние на скорость превращения КА (рис. 9a). На основании зависимости скорости реакции от температуры в координатах Аppeниуса (рис. 9б) определены кажущиеся энергии активации $E_{a}$ гидрирования КА (30 кДж/моль), а также образования бутаналя (34 кДж/моль) и КС (33 кДж/моль), которые имеют сопоста-

$$
-545-
$$




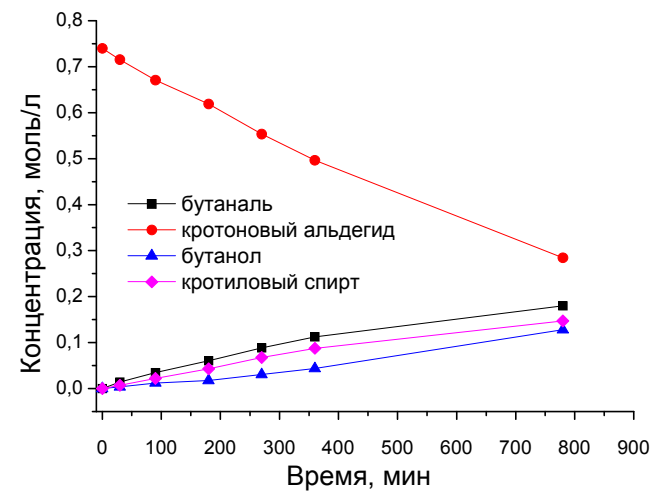

a)

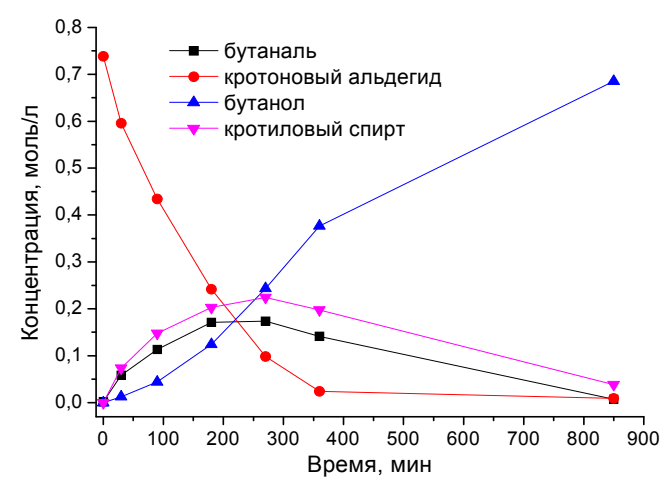

б)

Рис. 9. Кинетические зависимости для реакции гидрирования КА в присутствии: $\mathrm{a}-\mathrm{Re} / \mathrm{Al}_{2} \mathrm{O}_{3} ; 6-\mathrm{Ir} / \mathrm{Al}_{2} \mathrm{O}_{3}$. Условия реакции: $m_{\text {кат }}=150 \mathrm{Mг}, P\left(\mathrm{H}_{2}\right)=0,84 \mathrm{MПа}, T=453 \mathrm{~K}, C_{0}=0,74 \mathrm{M}$, растворитель: декан

Fig. 9. Kinetics of crotonaldehyde hydrogenation over a $-\mathrm{Re} / \mathrm{Al}_{2} \mathrm{O}_{3} ; \sigma-\mathrm{Ir} / \mathrm{Al}_{2} \mathrm{O}_{3}$. Reaction conditions: $m_{\text {cat }}=150$ $\mathrm{mg}, T=453 \mathrm{~K}, P\left(\mathrm{H}_{2}\right)=0.84 \mathrm{MPa}, C_{0}=0.74 \mathrm{M}$, solvent: decane

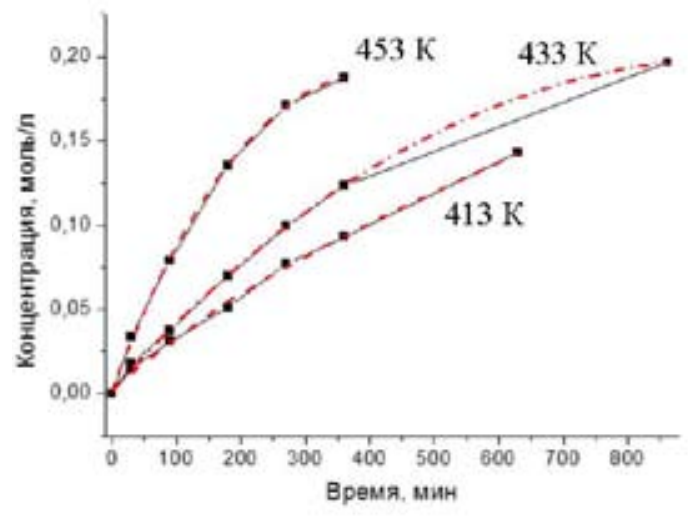

a)

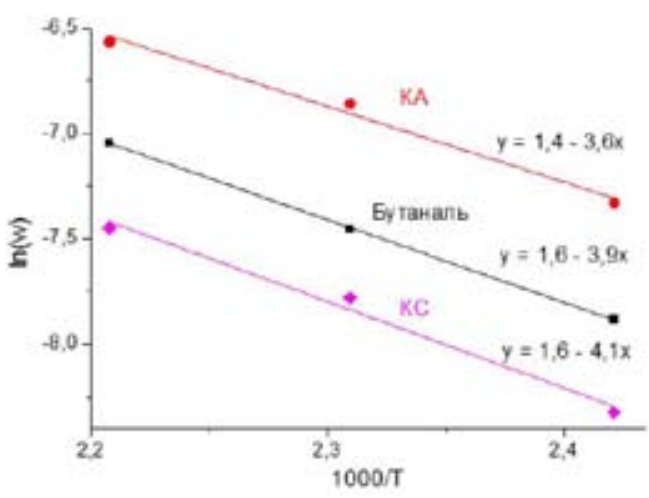

6)

Рис. 10. Экспериментальная зависимость концентрации КС от времени реакции (сплошная линия) и полиномиальная зависимость (пунктирная линия) (а), зависимость скорости реакции от температуры в координатах Аррениуса для КА, бутаналя и КС (б). Условия реакций: $m_{\text {кат }}\left(\mathrm{Ir} / \mathrm{Al}_{2} \mathrm{O}_{3}\right)=150$ мг, $P\left(\mathrm{H}_{2}\right)=0,84$ МПа, $T=413-453 \mathrm{~K}, C_{0}=0,74 \mathrm{M}$, растворитель: декан

Fig. 10. Dependence of crotyl alcohol concentration on time at 413, 433 and $453 \mathrm{~K}$ - experimental (solid line) and polynomial (dash line) (a), Arrhenius temperature dependence for crotonaldehyde, crotyl alcohol and butanal (б). Reaction conditions: $m_{\text {cat }}\left(\mathrm{Ir} / \mathrm{Al}_{2} \mathrm{O}_{3}\right)=150 \mathrm{mg}, P\left(\mathrm{H}_{2}\right)=0.84 \mathrm{MPa}, T=413-453 \mathrm{~K}, C_{0}=0,74 \mathrm{M}$, solvent: decane

вимые значения. Интересно отметить, что величина $E_{a}$ для гидрирования КА на $\mathrm{Ir} / \mathrm{Al}_{2} \mathrm{O}_{3}$ coпоставима с литературными данными, полученными для $\mathrm{Ir} / \mathrm{SiO}_{2}(20 \div 25$ кДж/моль) и $\mathrm{Ir} / \mathrm{TiO}$

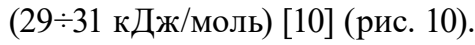

Была также изучена стабильность работы катализатора 4 \% $\mathrm{Ir} / \mathrm{Al}_{2} \mathrm{O}_{3}$ на трех циклах с длительностью цикла 1 ч. Показано, что селективность образования КС уменьшается, а селективность по бутаналю возрастает с каждым циклом (рис. 11). 


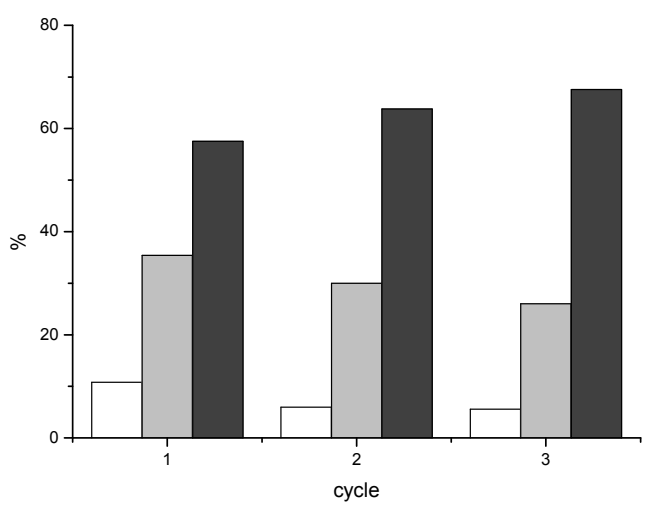

Рис. 11. Зависимость конверсии КА (белый цвет), селективности по КС (серый) и по бутаналю (черный) для катализатора $4 \% \mathrm{Ir} / \mathrm{Al}_{2} \mathrm{O}_{3}$ от количества циклов. Условия реакций: $m_{\text {кат }}=150$ мг, $P\left(\mathrm{H}_{2}\right)=0,84 \mathrm{MПа}$, $T=453 \mathrm{~K}, C_{0}=0,74 \mathrm{M}$, растворитель: декан

Fig. 11. Croton aldehyde conversion (white), and selectivity to crotyl alcohol (grey) and butanal (black) for different catalytic cycles. Reaction conditions: $m_{\text {cat }}\left(4 \% \mathrm{Ir} / \mathrm{Al}_{2} \mathrm{O}_{3}\right)=150 \mathrm{mg}, P\left(\mathrm{H}_{2}\right)=0.84 \mathrm{MPa}, T=453 \mathrm{~K}, C_{0}=0.74 \mathrm{M}$, solvent: decane

Таким образом, сравнительное исследование жидкофазного гидрирования КА в неполярных и полярных средах на монометаллических Pd-, Pt-, Ir-, Re-катализаторах, нанесенных на мезопористые носители - углеродный носитель Сибунит и $\gamma-\mathrm{Al}_{2} \mathrm{O}_{3}$, показало, что наиболее эффективно образование КС происходит при гидрировании КА на $\mathrm{Ir} / \mathrm{Al}_{2} \mathrm{O}_{3}$ в апротонном неполярном растворителе (декан). Селективность по КС возрастает с увеличением температуры, достигая $30 \%$ при конверсии $25 \%$ (453 K, 0,84 МПа). Найденная величина $E_{a}(\mathrm{KA})$ на $\mathrm{Ir} / \mathrm{Al}_{2} \mathrm{O}_{3}$ сопоставима с $E_{a}(\mathrm{KA})$ на $\mathrm{Ir} / \mathrm{TiO}_{2}$, проявляющем сильное взаимодействие металл-носитель. В ходе гидрирования КА на $\mathrm{Ir} / \mathrm{Al}_{2} \mathrm{O}_{3}$ на рециклах происходит его дезактивация, сопровождающаяся заметным снижением селективности по КС.

Авторы благодарны сотрудникам ИК СО РАН к.ф.-м.н. Е.Ю. Герасимову за исследование образцов методом ПЭМ, к.х.н. И.П. Просвирину за исследование образцов методом РФЭС, к.х.н. Н.В. Штерцер за исследование образцов методом ТГ-ДТА и И.Л. Краевской за исследование образцов рентгенофлюоресцентным методом анализа.

Работа выполнена при финансовой поддержск Министерства науки и высшего образования Российской Федерации (проект АААA-A17-117041710075-0).

Работа в части синтеза и анализа катализаторов поддержана грантом РФФИ о 18-53-45013 ИНД_а.

\section{Список литературы}

1. Li B., Hong X., Lin J.-J., Hu G.-S., Yu Q., Wang Y.-J., Luo M.-F., Lu J.-Q. Promoting effect of Ir on the catalytic property of $\mathrm{Ru} / \mathrm{ZnO}$ catalysts for selective hydrogenation of crotonaldehyde. Applied Surface Science 2013. Vol. 280, P. 179-185.

2. Narayanan S. Selective hydrogenation of unsaturated aldehydes and ketones. Bulletin of the Catalysis Society of India 2003. Vol. 2, P. 107-121.

$$
-547-
$$


3. Gallezot P., Richard D. Selective hydrogenation of $\alpha, \beta$-unsaturated aldehydes. Catalysis Reviews 1998. Vol. 40, P. 81-126.

4. Deliy I.V., Simakova I.L., Ravasio N., Psaro R. Catalytic behaviour of carbon supported platinum group metals in the hydrogenation and isomerization of methyl oleate. Applied Catalysis A: General 2009. Vol. 357(2), P. 170-177.

5. Simakova O.A., Simonov P.A., Romanenko A.V., Simakova I.L. Preparation of Pd/C catalysts via deposition of palladium hydroxide onto Sibunit carbon and their application to partial hydrogenation of rapeseed oil. Reaction Kinetics Catalysis Letters 2008. Vol. 95, P. 3-12.

6. Basale R. Selective catalytic hydrogenation of $\alpha, \beta$-unsaturated aldehyde to unsaturated alcohol: investigation of the role of the promoter. - Eindhoven: Technische Universiteit Eindhoven, 2013. P. 157.

7. Maki-Arvela P., Ha' jek J., Salmi T., Murzin D.Yu. Chemoselective hydrogenation of carbonyl compounds over heterogeneous catalysts. Applied Catalysis A: General 2005. Vol. 292, P. 1-49.

8. Hidalgo-Carrillo J., Aramendia M.A., Marinas A., Marinas J.M., Urbano F.J. Support and solvent effects on the liquid-phase chemoselective hydrogenation of crotonaldehyde over Pt catalysts. Applied Catalysis A: General 2010. Vol. 385, P. 190-200.

9. Gebauer-Henke E., Farbotko J., Touroude R., Rynkowski J. A comparative study of $\mathrm{Ir} / \mathrm{Ga}_{2} \mathrm{O}_{3}$, $\mathrm{Pt} / \mathrm{Ga}_{2} \mathrm{O}_{3}$, and $\mathrm{Ru} / \mathrm{Ga}_{2} \mathrm{O}_{3}$ catalysts in selective hydrogenation of crotonaldehyde. Kinetics and Catalysis 2008. Vol. 49(4), P. 574-580.

10. Reyes P., Aguirre M.C., Pecchi G., Fierro J.L.G. Crotonaldehyde hydrogenation on Ir supported catalysts. Journal of Molecular Catalysis A: Chemical 2000. Vol. 164, P. 245-251.

11. Englisch M., Ranade V.S., Lercher J.A. Liquid phase hydrogenation of crotonaldehyde over $\mathrm{Pt} / \mathrm{SiO}_{2}$ catalysts. Applied Catalysis A: General 1997. Vol. 167, P. 111-122.

12. He S., Xie L., Che M., Chan H.C., Yang L., Shi Z., Tang Y., Gao Q. Chemoselective hydrogenation of $\alpha, \beta$-unsaturated aldehydes on hydrogenated MoOx nanorods supported iridium nanoparticles. Journal of Molecular Catalysis A: Chemical 2016. Vol. 425, P. 248-254.

13. Ning X., Yu-Meng Xu Y.-M., Wu A.-Q., Tang C., Jia A.-P., Luo M.-F., Lu J.-Q. Kinetic study of selective hydrogenation of crotonaldehyde over Fe-promoted Ir/BN catalysts. Applied Surface Science 2019. Vol. 463, P. 463-473.

14. Tamura M., Tokonami K., Nakagawa Y., Tomishige K. Effective NbOx-modified $\mathrm{Ir} / \mathrm{SiO}_{2}$ catalyst for selective gas-phase hydrogenation of crotonaldehyde to crotyl alcohol. ACS Sustainable Chemistry and Engineering 2017. Vol. 5, P. 3685-3697.

15. Taniya K., Yu C.H., Takado H., Hara T., Okemoto A., Horie T., Ichihashi Y., Tsang S.C., Nishiyama S. Synthesis of bimetallic SnPt-nanoparticle catalysts for chemoselective hydrogenation of crotonaldehyde: Relationship between $\mathrm{Sn}_{\mathrm{x}} \mathrm{Pt}_{\mathrm{y}}$ alloy phase and catalytic performance. Catalysis Today 2018. Vol. 303, P. 241-248.

16. Ide M.S., Hao B., Neurock M., Davis R.J. Mechanistic insights on the hydrogenation of $\alpha, \beta$ unsaturated ketones and aldehydes to unsaturated alcohols over metal catalysts. ACS Catalysis 2012. Vol. 2, P. 671-683.

17. Mäki-Arvela P., Kuusisto J., Sevilla E.M., Simakova I., Mikkola J.-P., Myllyoja J., Salmi T., Murzin D.Yu. Catalytic hydrogenation of linoleic acid to stearic acid over different Pd- and Rusupported catalysts. Applied Catalysis A: General 2008. Vol. 345(2), P. 201-212. 
18. Zaytseva Y.A., Panchenko V.N., Simonov M.N., Shutilov A.A., Zenkovets G.A., Renz M., Simakova I.L., Parmon V.N. Effect of gas atmosphere on catalytic behaviour of zirconia, ceria and ceriazirconia catalysts in valeric acid ketonization. Topics in Catalysis 2013. Vol. 56(9-10), P. 846-855.

19. Mäki-Arvela P., Martin G., Simakova I., Tokarev A., Wärnå J., Hemming J., Holmbom B., Salmi T., Murzin D.Yu. Kinetics, catalyst deactivation and modeling in the hydrogenation of $\beta$-sitosterol to $\beta$-sitostanol over microporous and mesoporous carbon supported Pd catalysts. Chemical Engineering Journal 2009. Vol. 154(1-3), P. 45-51.

20. Godina L.I., Tokarev A.V., Simakova I.L., Mäki-Arvela P., Kortesmäki E., Gläsel J., Kronberg L., Etzold B., Murzin D.Y. Aqueous-phase reforming of alcohols with three carbon atoms on carbon-supported Pt. Catalysis Today 2018. Vol. 301, P. 78-89.

21. Simakova I.L., Demidova Y.S., Prikhod'ko S.A., Simonov M.N., Shabalin A.Yu. Liquid phase pentanol Guerbet-Markovnikov condensation over VIII group metals. Journal of Siberian Federal University. Chemistry 2016. Vol. 9 (2). P. 201-211.

22. Panchenko V.N., Paukshtis E.A., Simakova I.L., Murzin D.Yu. Solid base assisted n-pentanol coupling over VIII group metals: elucidation of the Guerbet reaction mechanism by DRIFTS. Industrial \& Engineering Chemistry Research 2017. Vol. 56(45), P. 13310-13321.

23. Simakova I.L., Demidova Y.S., Prikhod'ko S.A., Simonov M.N., Shabalin A.Yu. Catalytic hydrogenation of pentanoic acid over VIII group metals. Journal of Siberian Federal University. Chemistry 2015. Vol. 8 (3), P. 439-449. 